\title{
Face à l'incertitude : humilité, curiosité et partage
}

\author{
Facing uncertainty: humility, curiosity and sharing
}

«Faire de l'interruption, un nouveau chemin, faire de la chute, un pas de danse, faire de la peur, un escalier, du rêve, un pont, de la recherche... une rencontre.»

La notion d'incertitude, les façons de la gérer, voire de l'enseigner, font partie depuis longtemps du paysage des sciences de la santé. Il existe néanmoins une certaine réticence dans nos milieux cliniques et d'enseignement à la reconnaître et à l'aborder directement [1]. Pourtant, de nombreuses recherches ont mis en évidence les conséquences néfastes consécutives à la difficulté de gérer l'incertitude, que ce soit sur les soignant(e)s eux/elles-mêmes (risque de burnout), leur manière de raisonner (biais potentiels), de communiquer (difficultés relationnelles), d'utiliser les ressources à disposition, ou encore relatives à la sécurité et au bien-être des patient(e)s [2-5].

C'est à partir de ce constat que Baptiste Motte et al. [6] se sont lancés dans un travail de recherche conséquent et extrêmement bien documenté ayant pour objectif d'élaborer un cadre conceptuel approprié pour mieux comprendre et définir la notion d'incertitude, afin de pouvoir ensuite la «penser en tant qu'objet de formation». Une revue de la littérature leur a tout d'abord permis de faire une synthèse chronologique des nombreuses définitions de l'incertitude. Ce processus a mis en évidence l'évolution intégrative de ces différentes définitions au fil des ans, et leur a permis d'identifier la taxonomie de Han et al. [7] comme étant la mieux à même de décrire «clairement la diversité des incertitudes rencontrées par le professionnel de santé ou le patient dans le soin ». Pour ces auteurs, il est en effet important de considérer que l'incertitude résulte non seulement des caractéristiques du savoir médical, mais aussi de la façon dont il est mis en ouvre dans le cadre de la relation soignant-patient.

En d'autres termes, plus le/la soignant(e) accepte l'idée que les problèmes de santé requièrent des solutions qu'il faut construire dans le cadre d'une pratique soignante, d'un processus évolutif et contextualisé, plus il/elle sera à même de tolérer et gérer l'incertitude. À l'inverse, plus il/elle reste convaincu(e) que la connaissance est absolue et certaine, plus il lui sera difficile de développer des stratégies de gestion de l'incertitude.
Dans cette perspective, les auteurs soulignent que les médecins généralistes sont tout particulièrement confrontés à l'incertitude. Rencontrant en permanence «des patients aux demandes multiples et variées, présentant des symptômes isolés et peu spécifiques», ou encore souffrant de multi-morbidités nécessitant de revisiter régulièrement la priorisation des démarches de soins en accord avec eux et leurs proches [8], les médecins généralistes, mais aussi tous les soignant(e)s impliqués dans ce genre de pratique clinique, sont en effet au cœur de la gestion de l'incertitude.

Se recentrant sur la dimension pédagogique et sur la potentielle formation à l'incertitude, le propos de cette thèse devient particulièrement intéressant lorsque les auteurs démontrent comment la gestion de l'incertitude ne « dépend pas seulement des savoirs que l'on est capable de mobiliser, mais aussi de la façon dont on constitue et mobilise ces savoirs ». Les auteurs nous familiarisent donc avec la notion d'épistémologie personnelle, qui désigne les croyances que nous avons sur ce qu'est la connaissance et la manière avec laquelle nous mettons en application ces connaissances.

Dès lors, ils postulent que «plus les épistémologies personnelles d'un étudiant ou d'un praticien sont riches, plus il dispose de façons différentes et complémentaires d'appréhender l'incertitude dans la richesse de ses causes et de ses problématiques».

Les auteurs démontrent ensuite, au travers de différents exemples concrets, comment et dans quels contextes ces épistémologies peuvent évoluer et s'enrichir. C'est le cas notamment lorsque le/la soignant(e), face à une situation donnée, est confronté(e) aux limites de ses croyances, et s'engage ainsi dans un processus permettant de les faire évoluer.

Cet enrichissement favorise une «vision de la connaissance où il n'existe plus de vérité définitive mais où la connaissance est à construire activement par l'individu, dans une démarche réflexive, à partir de différents points de vue dont il évalue les niveaux de preuve et de pertinence».

Les auteurs mettent ensuite cette construction de la connaissance en lien avec l'approche pragmatiste de Dewey $[9,10]$, qui se conçoit comme un processus 
pédagogique d'enquête, réflexif et collectif, «au cours duquel on mobilise et on construit des connaissances permettant de cerner et de résoudre les problèmes issus des situations de soin ».

Ils présentent enfin comment cette approche peut se concrétiser dans l'enseignement en la contextualisant dans les modèles de soins actuels, qui mobilisent la dimension interdisciplinaire et dans lesquels le/la patient(e) est partenaire des soins.

Les auteurs concluent leur propos en faisant allusion à la pandémie de SARS-CoV-2; cette pandémie représente en effet un formidable prototype, quasi de laboratoire, de l'apparition soudaine et à l'état brut d'une incertitude scientifique, puis de la manière dont elle a été gérée, individuellement ou collectivement.

Le choix des auteurs de privilégier la taxonomie de Han pour décrire l'incertitude se vérifie ici particulièrement. La première source d'incertitude réside dans sa probabilité, c'est-à-dire le fait que l'évolution d'un phénomène observé reste indéterminée. Et c'est bien le cas avec la COVID-19; il ne passe pas un jour sans qu'une probabilité ne soit évoquée dans le cadre de cette pandémie: probabilité de virulence, de contagiosité, de gravité, d'évolution, de deuxième vague, pour n'en citer que quelques-unes. La deuxième source d'incertitude concerne l'ambiguïté: le manque de fiabilité des données scientifiques à disposition autour de ce virus a conduit à la diffusion d'informations inadéquates et de recommandations contradictoires. Enfin, la troisième source d'incertitude est relative à la complexité: la pathogénie, les mécanismes d'infection, les facteurs de risque ou de protection face à ce virus sont autant d'exemples de mécanismes complexes rendant la pathologie difficile à appréhender, de même que les effets de divers traitements testés.

Cette incertitude s'applique également sur la dimension des problématiques de Han, tant scientifique concernant la démarche diagnostique et thérapeutique, que pratique sur l'organisation des soins, et personnelle sur les enjeux de la maladie et de leurs traitements sur les patient(e)s et leur entourage.

La problématique scientifique est ici bien représentée par le raisonnement clinique, non seulement diagnostique mais également de prise en charge. La représentation mentale des connaissances au sujet de cette maladie est amenée à changer régulièrement, et les scripts $[11,12]$ qui y sont liés doivent évoluer rapidement. Les approches permettant parfois de limiter ce type d'incertitude - telles que l'approche probabiliste bayésienne fondée sur une probabilité clinique de maladie évoluant en fonction du résultat de certains tests dont on connaît les caractéristiques (sensibilité et spécificité notamment) - ne rassurent pas davantage, tant la probabilité de maladie a fluctué au gré de la pandémie dans une région donnée, et tant les tests (frottis à divers niveaux du tractus respiratoire, sérologies, radiologie, etc.) ont démontré leurs limites au fil du temps. C'est dire si le terrain est propice à toute une série de biais cognitifs [13], illustrés par de nombreux exemples cliniques, qu'il conviendrait de préciser par de futures recherches.
La présentation quelque peu statique d'une taxonomie ne doit néanmoins pas faire oublier les aspects dynamiques, notamment les interactions entre le niveau d'incertitude objective et la perception subjective de cette incertitude. Ces aspects sont surtout décrits pour l'incertitude diagnostique, alors que les aspects pronostiques ne devraient également pas être oubliés dans cette analyse $[14,15]$.

À cet égard, la problématique personnelle et la dimension du locus de Han méritent toute notre attention. En effet, si l'incertitude scientifique est bien objective, les réactions et les manières d'y faire face ont été, et sont encore très diverses. Du côté clinique, nous avons pu observer la difficulté du soin de la COVID-19 dans ces situations particulières, comment cette incertitude était présente dans l'esprit du clinicien ou de la clinicienne, et dans celui du patient ou de la patiente. Par ailleurs, et ce n'est pas anodin, de nombreux soignant(e)s ont aussi témoigné de cette incertitude pour eux/elles-mêmes, ou leurs proches, tant ils/elles avaient le souci de ne pas être infecté(e)s, voire de transmettre cette maladie à leur famille. Ceci s'est par exemple manifesté concrètement par une présence diminuée au chevet des patient(e)s, ou encore par un examen clinique abrégé.

Du côté des individus, des collectivités au sein de la société, les nombreuses réactions, parfois extrêmes et polarisées, témoignent dans leur diversité de cette difficulté de conception et de gestion des différentes facettes de l'incertitude. En fait, si depuis le début de cette pandémie, les soignant(e)s travaillent à affiner quotidiennement leurs scripts cliniques sur la COVID 19, c'est-àdire à développer et organiser un réseau de connaissances intégrant des aspects relatifs au diagnostic, aux investigations et aux aspects thérapeutiques, de la même manière, la société dans son ensemble va devoir, elle aussi, développer sa compréhension de cette maladie, de ses conséquences, et inventer une nouvelle manière de vivre et de se comporter en présence de ce virus, en apprenant à tolérer les incertitudes qui y sont reliées.

Devant l'ampleur du phénomène touchant à l'épistémologie personnelle, la confiance de la société prend le risque de se transformer en défiance envers le monde scientifique, un thème souvent commenté dans la presse par Michel Dubois, sociologue des sciences [16]. Cette perspective a incité plus de 2400 chercheurs à publier récemment une tribune commune d'opinion visant à fournir aux citoyens «un éclairage rationnel, mesuré, argumenté, compréhensible et humble, selon les codes de déontologie médicale, face à ceux qui veulent faire croire que l'intuition ou le "bon sens" médical seraient suffisants [17]». Il s'agit ici de rétablir l'éthique vis-à-vis des patient(e)s, en prenant en compte de manière globale l'éthique de la recherche et celle de l'approche clinique [18], plutôt que d'opposer ces aspects comme certains ont tenté de le faire. Se pose ici l'importance de la confiance que les clinicien(ne)s doivent avoir vis-à-vis de leurs sources de connaissances, mais aussi la capacité de les analyser et de les critiquer. 
C'est aussi l'occasion d'évoluer dans notre communication avec les patient(e)s au sujet de l'incertitude. Tout en gardant l'empathie et le souci du patient, nous avons l'opportunité de migrer vers une approche où ce dernier ou cette dernière devient partenaire, capable de contribuer aux décisions en ayant connaissance des éléments les plus probants possibles mais aussi de l'incertitude qui les entoure $[19,20]$. En cela, l'approche formalisée par le courant de l'evidence-based medicine (EBM) ne s'oppose pas à l'approche de soin mais la sert dans une relation de partenariat et de partage de l'information.

Enfin, pour faire le lien avec la volonté première des auteurs de proposer des points de repère et des pistes pour la formation des médecins, nous sommes quant à nous également convaincus que la formation doit inclure l'expérience de l'incertitude, la manière de la diminuer si faire se peut, puis de la tolérer et de la gérer. Ce processus d'apprentissage ne peut se réaliser que si cette expérience est accompagnée de supervision bienveillante, apportant à la fois la rétroaction nécessaire et le soutien légitimant sa présence comme une partie intégrante de nos vies de clinicien(e)s. L'enjeu peut être important puisque l'apprentissage de stratégies permettant de faire face à l'anxiété et au stress contribue à prédire le succès de la carrière médicale [21].

Ceci ne peut devenir naturel que si notre culture médicale, soignante, mais aussi collective, change, migrant du dogmatisme médical et de la seule responsabilité individuelle, vers une ouverture et un partage. En cela, le travail d'équipe et l'inter-professionnalité sont des ingrédients essentiels à améliorer, le partage de l'incertitude devenant ainsi un élément normal de toute décision.

Intégrer l'incertitude dans le processus plutôt que vouloir l'éviter ou la nier, considérer qu'elle fait partie intégrante du problème clinique à résoudre, sont des approches pouvant permettre d'apprendre à vivre en sa compagnie. Il s'agira ainsi peut-être de la prochaine révolution en médecine: tolérer l'incertitude [22]!

Et pour ce faire, que l'on soit clinicien(ne) ou enseignant(e), une posture, un chemin se dessinent... Humilité, curiosité et partage.

\section{Marie-Claude AUDÉTAT ${ }^{1,2,4, *}$ Mathieu NENDAZ ${ }^{1,3, *}$}

${ }^{1}$ Unité de développement et de recherche en éducation médicale (UDREM), Faculté de médecine, Université de Genève, Genève, Suisse

${ }^{2}$ Unité des internistes généralistes et pédiatres (UIGP), Faculté de médecine, Université de Genève, Genève, Suisse

${ }^{3}$ Département de médecine, Faculté de médecine, Université de Genève, Genève, Suisse ${ }^{4}$ Départementde médecine de famille et de médecine d'urgence DMFMU, Faculté de médecine, Université de Montréal, Montréal, Canada

* Mailto: Marie-Claude.Audetat@unige.ch et Mathieu.Nendaz@unige.ch.

\section{Références}

1. Logan R, Scott P. Uncertainty in clinical practice: implications for quality and costs of health care. Lancet 1996;347:595-8.

2. Kruglanski A, Webster D. Motivated closing of the mind: "seizing" and "freezing". Psychol Rev 1996;103:263-83.

3. Cooke G, Doust J, Steele M. A survey of resilience, burnout, and tolerance of uncertainty in Australian general practice registrars. BMC Med Educ 2013;13:1-6.

4. Bhise V, Meyer A, Menon S, Singhal G, Street R, Giardina $\mathrm{T}$, et al. Patient perspectives on how physicians communicate diagnostic uncertainty: an experimental vignette study. Int J Qual Health Care 2018;30:2-8.

5. Simpkin AL, Khan A, West DC, Garci BM, Sectish TC, Spector ND, et al. Stress from uncertainty and resilience among depressed and burned out residents: a cross-sectional study. Acad Pediatr 2018;18:698-704

6. Motte B, Aiguier G, Van Pee D, Cobbaut J-P. Mieux comprendre l'incertitude en médecine pour former les médecins. Pédagogie Médicale 2020;21: doi: 10.1051/ pmed/2020025.

7. Han PKJ, Klein WMP, Arora NK. Varieties of uncertainty in health care: a conceptual taxonomy. Med Decis Making 2011;31:828-38.

8. Audétat M-C, Sader J, Cairo Notari S, Ritz C, Caire Fon N, Maisonneuve $\mathrm{H}$, et al. Understanding and promoting clinical reasoning in chronic and multimorbid conditions: a call for GPs and healthcare professionals. Health 2019;11:1338-46.

9. Schön DA. À la recherche d'une nouvelle épistémologie de la pratique et de ce qu'elle implique pour l'éducation des adultes. Paris: Presses universitaires de France, 2011.

10. Dewey J. Logique. La théorie de l'enquête. Genèses sciences sociales et histoire 1994;17:168-9.

11. Charlin B, Boshuizen HP, Custers EJ, Feltovich PJ. Scripts and clinical reasoning. Med Educ 2007;41:1178-84.

12. Lubarsky S, Dory V, Audétat M-C, Custers E, Charlin B. Using script theory to cultivate illness script formation and diagnostic reasoning in health professions education. Can Med Educ J 2015;6(2):e61-e70.

13. Nendaz M, Perrier A. Diagnostic errors and flaws in clinical reasoning: mechanisms and prevention in practice. Swiss Med Wkly 2012;142:w13706.

14. Santhosh L, Chou CL, Connor DM. Diagnostic uncertainty: from education to communication. Diagnosis (Berl) 2019;6:121-6.

15. Smith AK, White DB, Arnold RM. Uncertainty: the other side of prognosis. N Engl J Med 2013;368:2448-50.

16. Dubois M. Le coronavirus peut-il altérer la confiance en la science ? 2020 [On-line]. Disponible sur : https://lejournal. cnrs.fr/billets/le-coronavirus-peut-il-alterer-la-confianceen-la-science.

17. Collectif. Lutte contre le Covid-19 : la médecine ne relève pas d'un coup de poker. Le Temps 15 juin 2020 [On-line]. Disponible sur : https://www.letemps.ch/opinions/luttecontre-covid19-medecine-ne-releve-dun-coup-poker.

18. Coutellec L. Penser l'indissociabilité de l'éthique de la recherche, de l'intégrité scientifique et de la responsabilité sociale des sciences. Clarification conceptuelle, propositions épistémologiques. Revue d'anthropologie des connaissances 2019;13:381-98

19. Lussier M, Richard C. En l'absence de panacée universelle, Répertoire des relations médecin-patient. Le médecin de famille canadien 2008;54:1096-99.

20. Karazivan P, Dumez V, Flora L, Pomey M, Del Grande C, Ghadiri D, et al. The patient-as-partner approach in health 
care: a conceptual framework for a necessary transition. Acad Med 2015;90:437-41

21. Tartas M, Walkiewicz M, Budzinski W, Majkowicz M, Wójcikiewicz K, Zdun-Ryz ewska A. The coping strategies during medical education predict style of success in medical career: a 10-year longitudinal study. BMC Med Educ 2016;16:186.

22. Simpkin AL, Schwartzstein RM. Tolerating uncertainty — The next medical revolution? N Engl J Med 2016;375: 1713-5. 